\title{
FEEDBACK STABILIZATION OF A SIMPLIFIED MODEL OF FLUID-STRUCTURE INTERACTION ON A TREE
}

\author{
KAÏS AMMARI, FARHAT SHEL, AND MUTHUSAMY VANNINATHAN
}

\begin{abstract}
In this paper we study the dynamic feedback stability for a simplified model of fluid-structure interaction on a tree. We prove that, under some conditions, the energy of the solutions of the system decay exponentially to zero when the time tends to infinity. Our technique is based on a frequency domain method and a special analysis for the resolvent.
\end{abstract}

\section{Contents}

1. Introduction 2

2. Wellposedness 5

3. Exponential stability 6

4. Some other cases 10

4.1. A circuit

4.2. A start with two fixed endpoints 14

5. A chain with non equal mass points

Acknowledgment

References

2010 Mathematics Subject Classification. 35B40, 35L05, 93D15.

Key words and phrases. Dynamic feedback, asymptotic stabilization, resolvent method. 


\section{Introduction}

First of all, we introduce some notations needed to formulate the problem under consideration, as introduced in [1] or in [12]. Let $\mathcal{T}$ be a tree (i.e. a planar connected graph without closed paths). By the multiplicity of a vertex of $\mathcal{T}$ we mean the number of edges that branch out from the vertex. If the multiplicity is equal to one, the vertex is called exterior; otherwise, it is said to be interior. We denote by $\left\{e_{1}, \ldots, e_{N}\right\}$ the set of edges of $\mathcal{T}$ and $\left\{a_{1}, \ldots, a_{N+1}\right\}$ its set of vertices and we assume that $a_{1}$ is the root of $\mathcal{T}$ which will be denoted by $\mathcal{R}$, that $e_{1}$ is the edge containing $\mathcal{R}$ and $a_{2}$ is its vertex different from $\mathcal{R}$.

We denote by $\mathcal{M}$ the set of the interior vertices of $\mathcal{T}$ and by $\mathcal{S}$ the set of the exterior vertices, except $\mathcal{R}$ and denote $I_{\mathcal{M}}$ and $I_{\mathcal{S}}$ the sets of indices of interior and exterior vertices, except $\mathcal{R}$, respectively. Then $I=I_{\mathcal{M}} \cup I_{\mathcal{S}}$ is the set of indices of all vertices, except the root $\mathcal{R}$. We denote by $J$ the set $\{1, \ldots, N\}$ and for $k \in I$ we will denote by $J_{k}$ the set of indices of edges adjacent to $a_{k}$. If $k \in \mathcal{M}$, then the indice of the unique element of $J_{k}$ will be denoted by $j_{k}$.

Furthermore, the length of the edge $e_{j}$ will be denoted by $\ell_{j}$. Then $e_{j}$ may be parametrized by its arc length by means of the function $\pi_{j}:\left[0, \ell_{j}\right] \longrightarrow e_{j}, x_{j} \mapsto \pi_{j}\left(x_{j}\right)$, and sometimes identified with the interval $\left(0, \ell_{j}\right)$.

For a function $y: \mathcal{T} \longrightarrow \mathbb{C}$ we set $y^{j}=y \circ \pi_{j}$ its restriction to the edge $e_{j}$ and we will denote $y^{j}(x)=y^{j}\left(\pi_{j}(x)\right)$ for any $x$ in $\left(0, \ell_{j}\right)$.

The incidence matrix $D=\left(d_{k j}\right)_{(N+1) \times N}$ is defined by

$$
d_{k j}=\left\{\begin{array}{l}
1 \text { if } \pi_{j}\left(\ell_{j}\right)=a_{k}, \\
-1 \text { if } \pi_{j}(0)=a_{k}, \\
0 \text { otherwise }
\end{array}\right.
$$

We denote by $\langle.,$.$\rangle and \|$.$\| the inner product and norm in L^{2}$-space, respectively.

In this paper, we study the stability of a model of fluid propagating in a 1-d network, under some feedback forces applied at exteriors nodes, with the presence of point mass at inner nodes, see Figure 1. At rest, the network coincides with the tree $\mathcal{T}$.

More precisely, we consider the following initial and boundary value problem.

$$
\left\{\begin{array}{l}
y_{t t}^{j}-y_{x x}^{j}=0 \text { in }\left(0, \ell_{j}\right) \times(0, \infty), \quad j \in J, \\
\sum_{j \in J_{k}} d_{k j} y_{x}^{j}\left(a_{k}, t\right)=s_{k}^{\prime}(t), \quad k \in I_{\mathcal{M}}, \\
s_{k}^{\prime \prime}(t)+s_{k}(t)=-y_{t}\left(a_{k}, t\right), \quad k \in I_{\mathcal{M}}, \\
y^{j}\left(a_{k}, t\right)=y^{l}\left(a_{k}, t\right), \quad j, l \in J_{k}, \quad k \in I_{\mathcal{M}}, \\
y^{1}\left(a_{1}, t\right)=0, \\
d_{k j_{k}} y_{x}^{j_{k}}\left(a_{k}, t\right)=-y_{t}\left(a_{k}, t\right), \quad k \in I_{\mathcal{S}}, \\
s_{k}(0)=s_{k, 0}, \quad s_{k}^{\prime}(0)=s_{k, 1}, \quad k \in I_{\mathcal{M}}, \\
y^{j}(x, 0)=y_{0}^{j}(x), y_{t}^{j}(x, 0)=y_{k}^{1}(x), \quad x \in\left(0, \ell_{j}\right), j \in J,
\end{array}\right.
$$

where $y^{j}:\left[0, \ell_{j}\right] \times(0, \infty) \longrightarrow \mathbb{R}, j \in J$ represents the velocity potential of the fluid on the edge $e_{j}$ and $s_{k}:(0, \infty) \longrightarrow \mathbb{R}, k \in I_{\mathcal{M}}$ denotes the displacement of the point mass 


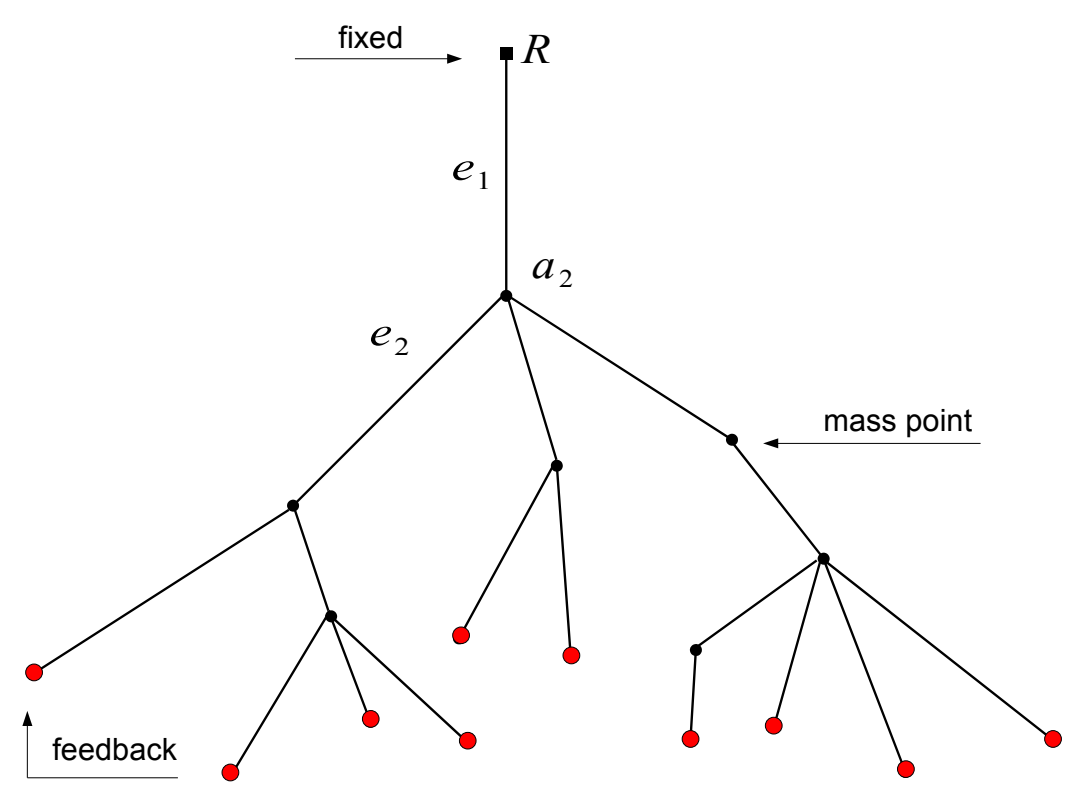

FiguRE 1. A tree-shaped network

occupying the node $a_{k}$. These functions allow us to identify the network with its rest graph. See [8] for more details.

This simplified model of fluid-structure interaction draws on the work of Ervedoza and Vanninathan [9]; they consider a fluid occupying a domain in two dimensions and a solid immersed in it and prove some results of controllability of such system; see also [16]. The problem of fluid structure interaction in one dimension has been studied by several authors. In [17] the authors study the asymptotic behavior of a one dimensional model of mass point moving in a fluid. They consider the same system in [18] but with a finite number of mass points floating in the fluid. Recently Tucsnak et al. [11] studied the controllability of a similar system.

Note that the point-wise (or boundary) stabilization on the wave equation has been treated during the last few years, see for example [2] for one string, and [3, 4, 5] for some networks of strings.

The main result of this paper asserts that, under some conditions, the energy of the solutions of the dissipative system decay exponentially to zero when the time tends to infinity. The method is based on a frequency domain method and a special analysis for the resolvent.

If $(y, s)=\left(\left(y^{j}\right)_{j \in J},\left(s_{k}\right)_{k \in \mathcal{M}}\right)$ is a solution of 1.1$)$ we define the energy of $(y, s)$ at instant $t$ by

$$
E(t)=\frac{1}{2} \sum_{j \in J} \int_{0}^{\ell_{j}}\left(\left|y_{t}^{j}(x, t)\right|^{2}+\left|y_{x}^{j}(x, t)\right|^{2}\right) d x+\frac{1}{2} \sum_{k \in I_{\mathcal{M}}}\left(\left|s_{k}^{\prime}(t)\right|^{2}+\left|s_{k}(t)\right|^{2}\right) .
$$


Simple formal calculations show that a sufficiently smooth solution of (1.1) satisfies the energy estimate

$$
E(0)-E(t)=\sum_{k \in I_{\mathcal{S}}} \int_{0}^{t}\left|y_{t}^{j_{k}}\left(a_{k}, s\right)\right|^{2} d s, \quad \forall t \geq 0 .
$$

In particular 1.2 implies that

$$
E(t) \leq E(0), \quad \forall t \geq 0 .
$$

Estimate above suggests that the natural wellposedness space for $(1.1)$ is

$$
H=V \times \prod_{j \in J} L^{2}\left(0, \ell_{j}\right) \times\left(\prod_{k \in I_{\mathcal{M}}} \mathbb{C}\right)^{2}
$$

where

$$
V=\left\{\Phi \in \prod_{j \in J} H^{1}\left(0, \ell_{j}\right), \Phi^{1}(\mathcal{R})=0, \Phi^{j}\left(a_{k}\right)=\Phi^{l}\left(a_{k}\right), j, l \in J_{k}, k \in I_{\mathcal{M}}\right\} .
$$

We can rewrite the system (1.1) as a first order differential equation, by putting $z(t)=\left(\begin{array}{c}y(t) \\ y^{\prime}(t) \\ s(t) \\ s^{\prime}(t)\end{array}\right)$

$$
z^{\prime}(t)=\mathcal{A} z(t), \quad z(0)=z_{0}=\left(\begin{array}{c}
y_{0} \\
y_{1} \\
s^{0} \\
s^{1}
\end{array}\right)
$$

where

$$
\mathcal{A}\left(\begin{array}{c}
y \\
v \\
p \\
q
\end{array}\right)=\left(\begin{array}{c}
v \\
\frac{d^{2} y}{d x^{2}} \\
q \\
-p-v_{\mathcal{M}}
\end{array}\right), \forall\left(\begin{array}{c}
y \\
v \\
p \\
q
\end{array}\right) \in \mathcal{D}(\mathcal{A})
$$

with $v_{\mathcal{M}}=\left(v\left(a_{k}\right)\right)_{k \in I_{\mathcal{M}}}$, and

$$
\left.\mathcal{D}(\mathcal{A})=\left\{(y, v, p, q) \in\left[\prod_{j \in J} H^{2}\left(0, \ell_{j}\right) \cap V\right] \times V \times\left(\prod_{k \in I_{\mathcal{M}}} \mathbb{C}\right)^{2} \text { satisfying } 1.3\right)\right\}
$$

where

$$
\left\{\begin{array}{l}
\sum_{j \in J_{k}} d_{k j} \frac{d y^{j}}{d x}\left(a_{k}\right)=q_{k}, \forall k \in I_{\mathcal{M}}, \\
d_{k j} \frac{d y^{j_{k}}}{d x}\left(a_{k}\right)=-v^{j_{k}}\left(a_{k}\right), \forall k \in I_{\mathcal{S}} .
\end{array}\right.
$$

To simplify the notations, sometimes, we will write $y\left(a_{k}\right)$ instead of $y^{j}\left(a_{k}\right)$ for $y$ in $V$.

The outline of this work is the following. In Section 2 we prove the existence and uniqueness of solutions for system (1.1). Section 3 is devoted to prove the exponential stability of the associated semi group. Finally, in Section 4, we prove the lack of exponential stability if the graph contain a circuit or if there is at least one uncontrolled 
exterior node (other than the root). The section 5 is devoted to the study of the case of a chain with non equal mass points.

\section{Wellposedness}

Lemma 2.1. The operator $\mathcal{A}$ generates a $\mathcal{C}_{0}$-semigroup of contractions $(S(t))_{t \in \mathbb{R}^{+}}$.

Proof. It is clear that the operator $\mathcal{A}$ is dissipative, moreover, for every $z=(y, v, p, q) \in$ $\mathcal{D}(\mathcal{A})$

$$
\operatorname{Re}\left(\langle\mathcal{A} z, z\rangle_{H}\right)=-\sum_{k \in I_{\mathcal{S}}}\left|v^{j_{k}}\left(a_{k}\right)\right|^{2} \leq 0
$$

Now we prove that every positive real number $\lambda$ belongs to $\rho(\mathcal{A})$, the resolvent set of $\mathcal{A}$. For this, let $Z=(f, g, c, d) \in H$ and we solve the equation

$$
(\lambda-\mathcal{A}) z=Z
$$

with $z$ in $\mathcal{D}(\mathcal{A})$.

We rewrite 2.5) explicitly

$$
\left\{\begin{array}{l}
\lambda y^{j}-v^{j}=f^{j}, \quad j \in J, \\
\lambda v^{j}-\frac{d^{2} y^{j}}{d x^{2}}=g^{j}, \quad j \in J, \\
\lambda p_{k}-q_{k}=c_{k}, \quad k \in I_{\mathcal{M}}, \\
\lambda q_{k}+p_{k}+v_{k}\left(a_{k}\right)=d_{k}, \quad k \in I_{\mathcal{M}} .
\end{array}\right.
$$

We eliminate $(v, q)$ in $(2.6)$ to get

$$
\begin{aligned}
\lambda^{2} y^{j}-\frac{d^{2} y^{j}}{d x^{2}} & =g^{j}+\lambda f^{j}, \quad j \in J, \\
\left(\lambda^{2}+1\right) p_{k}+v_{k}\left(a_{k}\right) & =d_{k}+\lambda c_{k}, \quad k \in I_{\mathcal{M}} .
\end{aligned}
$$

Let $w$ in $V$. Multiplying (2.7) by $w^{j}$ in $L^{2}\left(0, \ell_{j}\right)$ and summing over $j \in J$,

$$
\begin{aligned}
& \lambda^{2} \sum_{j \in J}\left(\int_{0}^{\ell_{j}} y^{j} \overline{w^{j}} d x+\int_{0}^{\ell_{j}} \frac{d y^{j}}{d x} \frac{d \overline{w^{j}}}{d x} d x\right)-\sum_{k \in I_{\mathcal{M}}} \overline{w\left(a_{k}\right)} q_{k}+\sum_{k \in I_{\mathcal{S}}} \overline{w\left(a_{k}\right)}\left(a_{k}\right) \\
(2.9)= & \sum_{j \in J} \int_{0}^{\ell_{j}}\left(g^{j}+\lambda f^{j}\right) \overline{w^{j}} d x .
\end{aligned}
$$

Multiplying (2.8) by $r_{k} \in \mathbb{C}$ and summing over $k \in I_{\mathcal{M}}$, we get

$$
\left(\lambda^{2}+1\right) \sum_{k \in I_{\mathcal{M}}} p_{k} \overline{r_{k}}+\sum_{k \in I_{\mathcal{M}}} v\left(a_{k}\right) \overline{r_{k}}=\sum_{k \in I_{\mathcal{M}}}\left(d_{k}+\lambda c_{k}\right) \overline{r_{k}} .
$$

Summing 2.9 and 2.10 we get

$$
\begin{array}{r}
\lambda^{2} \sum_{j \in J}\left(\left\langle y^{j}, w^{j}\right\rangle+\left\langle\frac{d y^{j}}{d x}, \frac{d w^{j}}{d x}\right\rangle\right)+\left(\lambda^{2}+1\right) \sum_{k \in I_{\mathcal{M}}} p_{k} \overline{r_{k}}+\sum_{k \in I_{\mathcal{M}}}\left(v\left(a_{k}\right) \overline{r_{k}}-\overline{w\left(a_{k}\right)} q_{k}\right) \\
+\sum_{k \in I_{\mathcal{S}}} \overline{w\left(a_{k}\right)} v\left(a_{k}\right)=\sum_{j \in J}\left\langle g^{j}+\lambda f^{j}, w^{j}\right\rangle+\sum_{k \in I_{\mathcal{M}}}\left(d_{k}+\lambda c_{k}\right) \overline{r_{k}}
\end{array}
$$


to obtain

$$
a((y, p),(w, r))=f(w, r)
$$

where

$$
\begin{aligned}
a((y, p),(w, r))= & \lambda^{2} \sum_{j \in J}\left(\left\langle y^{j}, w^{j}\right\rangle+\left\langle\frac{d y^{j}}{d x}, \frac{d w^{j}}{d x}\right\rangle\right)+\left(\lambda^{2}+1\right) \sum_{k \in I_{\mathcal{M}}} p_{k} \overline{r_{k}} \\
& +\lambda \sum_{k \in I_{\mathcal{M}}}\left(y\left(a_{k}\right) \overline{r_{k}}-\overline{w\left(a_{k}\right)} p_{k}\right)+\lambda \sum_{k \in I_{\mathcal{S}}} \overline{w\left(a_{k}\right)} y\left(a_{k}\right)
\end{aligned}
$$

and

$$
\begin{aligned}
f(w, r)= & \sum_{j \in J}\left\langle g^{j}+\lambda f^{j}, w^{j}\right\rangle+\sum_{k \in I_{\mathcal{M}}}\left(d_{k}+\lambda c_{k}\right) \overline{r_{k}}-\sum_{k \in I_{\mathcal{M}}} \overline{w\left(a_{k}\right)} c_{k} \\
& +\sum_{k \in I_{\mathcal{M}}} f\left(a_{k}\right) \overline{r_{k}}+\sum_{k \in I_{\mathcal{S}}} \overline{w\left(a_{k}\right)} f\left(a_{k}\right) .
\end{aligned}
$$

$a$ is a continuous sesquilinear form on $V \times \prod_{k \in I_{\mathcal{M}}} \mathbb{C}$ and $f$ is a continuous anti-linear form on $V \times \prod_{k \in I_{\mathcal{M}}} \mathbb{C}$. Moreover

$a((w, r),(w, r))=\lambda^{2} \sum_{j \in J}\left(\left\|w^{j}\right\|^{2}+\left\|\frac{d w^{j}}{d x}\right\|^{2}\right)+\left(\lambda^{2}+1\right) \sum_{k \in I_{\mathcal{M}}}\left|r_{k}\right|^{2}-2 \mathbf{i} I m\left(\sum_{k \in I_{\mathcal{M}}} \overline{w\left(a_{k}\right)} r_{k}\right)$

and then

$$
|a((w, r),(w, r))| \geq \lambda^{2}\left[\sum_{j \in J}\left(\left\|w^{j}\right\|^{2}+\left\|\frac{d w^{j}}{d x}\right\|^{2}\right)+\sum_{k \in I_{\mathcal{M}}}\left|r_{k}\right|^{2}\right]
$$

that is, $a$ is coercive. The conclusion result immediately from the Lax Milgram lemma.

Proposition 2.2. Suppose that $\left(y_{0}, y_{1}, s^{0}, s^{1}\right) \in H$. Then the problem (1.1) admits a unique solution

If $\left(y_{0}, y_{1}, s^{0}, s^{1}\right) \in \mathcal{D}(\mathcal{A})$ then

$$
\left(y, y^{\prime}, s, s^{\prime}\right) \in \mathcal{C}([0,+\infty) ; H)
$$

$$
\left(y, y^{\prime}, s, s^{\prime}\right) \in \mathcal{C}([0,+\infty), \mathcal{D}(\mathcal{A})) \cap \mathcal{C}^{1}([0,+\infty) ; \mathcal{H}) .
$$

Moreover $(y, s)$ satisfies the energy estimate (1.2).

\section{Exponential stability}

It is clear that if $\mathcal{T}$ contains an edge $e_{j}$, not attached to a leaf, with length $\ell_{j} \in \pi \mathbb{N}$ then $\mathbf{i}$ is eigenvalue of $\mathcal{A}$ with eigenvector $z=(y, v, p, q) \operatorname{such}$ that $y^{j}=\mathbf{i} \sin x, v^{j}=-\sin x$, $p_{k}=1$ and $q_{k}=\mathbf{i}$ when $a_{k}$ is the nearest end of $e_{j}$ to the root $\mathcal{R}$, and all the other compoents of $z$ are null.

In the following, the tree $\mathcal{T}$ is said to be a Pi-tree if it has has no edges of length $m \pi$ with $m \in \mathbb{N}^{*}$, except maybe those attached to leaves. Then we have the following result: 
Lemma 3.1. The spectrum of $\mathcal{A}$ contains no point on the imaginary axis if and only if $\mathcal{T}$ is a Pi-tree.

Proof. Since $\mathcal{A}$ has compact resolvent, its spectrum $\sigma(\mathcal{A})$ only consists of eigenvalues of $\mathcal{A}$. We will show that the equation

$$
\mathcal{A} z=\mathbf{i} \beta z
$$

with $z=\left(\begin{array}{c}y \\ v \\ p \\ q\end{array}\right) \in \mathcal{D}(\mathcal{A})$ and $\beta \in \mathbb{R}$ has only trivial solution.

By taking the inner product of (3.11) with $z \in H$ and using that

$$
\operatorname{Re}\left(\langle\mathcal{A} z, z\rangle_{H}\right)=-\sum_{k \in I_{\mathcal{S}}}\left|v\left(a_{k}\right)\right|^{2}
$$

we obtain that $v\left(a_{k}\right)=0$ for $k \in I_{\mathcal{S}}$ and then,

$$
\frac{d y}{d x}\left(a_{k}\right)=0 \text { for } k \in I_{\mathcal{S}} .
$$

Now the equation (3.11) can be rewritten explicitly as

$$
\begin{aligned}
v^{j} & =\mathbf{i} \beta y^{j}, \quad j \in J, \\
\frac{d^{2} y^{j}}{d x^{2}} & =\mathbf{i} \beta v^{j}, \quad j \in J, \\
q_{k} & =\mathbf{i} \beta p_{k}, \quad k \in I_{\mathcal{M}}, \\
-p_{k}-v\left(a_{k}\right) & =\mathbf{i} \beta q_{\kappa}, \quad k \in I_{\mathcal{M}} .
\end{aligned}
$$

If $\beta=0$ then $v=0, q=0$ and $p=0$.

Multiplying the second equation in the above system by $y^{j}$ and then summing over $j$, we obtain

$$
\sum_{j \in J}\left\|\frac{d y^{j}}{d x}\right\|^{2}=0,
$$

which implies, using continuity condition of $y$ at inner nodes and its Dirichlet condition at $\mathcal{R}$, that $y=0$.

Next, we suppose that $\beta \neq 0$. We have, using 3.13 .3 .16 ,

$$
\begin{aligned}
\beta^{2} y^{j}+\frac{d^{2} y^{j}}{d x^{2}} & =0, \quad j \in J, \\
\left(\beta^{2}-1\right) p_{k} & =v\left(a_{k}\right), \quad k \in I_{\mathcal{M}} .
\end{aligned}
$$

The function $y^{j}, j \in J$ is then of the form

$$
y^{j}=\alpha_{1} \sin (\beta x)+\alpha_{2} \cos (\beta x) .
$$

Using 3.12 and 3.13 we deduce that, for $k \in I_{\mathcal{S}}, y^{j_{k}}=0$ and then $v^{j_{k}}=0$.

For the sequel of the proof we consider two cases:

First case: $\beta^{2}=1$. From (3.18), (3.13) and the Dirichlet condition at $\mathcal{R}$, we deduce that $y^{j}(0)=y^{j}\left(\ell_{j}\right)=0$ for $j \in J$. Since $\mathcal{T}$ is a Pi-tree we conclude that $y^{j}=0$ for $j \in J$. Return back to the balance conditions and (3.15), one can deduce that $q=p=0$ and hence $y=0$. 
Second case: $\beta^{2} \neq 1$. Let $a_{k}$ the second end of an edge $e_{j}$ attached to a leaf. Then $p_{k}=0$ and $q_{k}=0$. Let $e_{l}$ the edge ended by $a_{k}$ and not attached to a leaf. We have $y^{l}\left(a_{k}\right)=0$ and $\frac{d y^{l}}{d x}\left(a_{k}\right)=0$. Then by $3.17 y^{l}=0$. We iterate such procedure from root to leaves to conclude that $y=0$.

The main result of this paper concerns the precise asymptotic behavior of the solution of (1.1). Our technique is based on a frequency domain method and a special analysis for the resolvent.

Recall that the system (1.1) is said to be exponentially stable if there exist two constants $M, \omega>0$, such that for all $\left(y_{0}, y_{1}, s^{0}, s^{1}\right) \in H$,

$$
E(t) \leq M e^{-\omega t}\left\|\left(y_{0}, y_{1}, s^{0}, s^{1}\right)\right\|_{H}^{2}, \quad \forall t \geq 0 .
$$

Then, our main result is the following:

Theorem 3.2. The system defined by equations (1.1) is exponentially stable if and only if $\mathcal{T}$ is a Pi-tree.

Proof. By classical result (see Huang [10] and Prüss [13]) it suffices to show that the corresponding operator $\mathcal{A}$ satisfies the following two conditions:

$$
\rho(\mathcal{A}) \supset\{\mathbf{i} \beta \mid \beta \in \mathbb{R}\} \equiv \mathbf{i} \mathbb{R},
$$

and

$$
\lim \sup _{|\beta| \rightarrow \infty}\left\|(\mathbf{i} \beta-\mathcal{A})^{-1}\right\|<\infty
$$

where $\rho(\mathcal{A})$ denotes the resolvent set of the operator $\mathcal{A}$.

By lemma 3.1 the condition (3.19) is satisfied. Suppose that the condition (3.20) is false. By the Banach-Steinhaus Theorem (see [7]), there exist a sequence of real numbers $\beta_{n} \rightarrow \infty\left(\beta_{n}>0\right.$ without loss of generality) and a sequence of vector $z_{n}=\left(\begin{array}{c}y_{n} \\ v_{n} \\ p_{n} \\ q_{n}\end{array}\right) \in$ $\mathcal{D}(\mathcal{A})$ with $\left\|z_{n}\right\|_{H}=1$ such that

$$
\left\|\left(\mathbf{i} \beta_{n} I-\mathcal{A}\right) z_{n}\right\|_{H} \longrightarrow 0 \quad \text { as } n \longrightarrow \infty
$$

i.e.

$$
\begin{aligned}
\mathbf{i} \beta_{n} y_{n}^{j}-v_{n}^{j} & \equiv f_{n}^{j} \longrightarrow 0 \quad \text { in } \quad H^{2}\left(0, \ell_{j}\right), \\
\mathbf{i} \beta_{n} v_{n}^{j}-\frac{d^{2} y_{n}^{j}}{d x^{2}} & \equiv g_{n}^{j} \longrightarrow 0 \quad \text { in } L^{2}\left(0, \ell_{j}\right), \\
\mathbf{i} \beta_{n} p_{k, n}-q_{k, n} & \equiv h_{k, n} \longrightarrow 0 \quad \text { in } \mathbb{C}, \\
\mathbf{i} \beta_{n} q_{k, n}+p_{k, n}+v_{n}\left(a_{k}\right) & \equiv r_{k, n} \longrightarrow 0 \quad \text { in } \mathbb{C} .
\end{aligned}
$$

Our goal is to derive from 3.21 that $\left\|z_{n}\right\|_{H}$ converge to zero, thus, a contradiction.

The proof is divided in three steps:

First step. Recall that for every $j$ in $J$,

$$
\frac{\left\|v^{j}\right\|_{\infty}}{\beta_{n}^{1 / 2}} \leq \frac{C_{1}}{\beta_{n}^{1 / 2}}\left\|\frac{d v^{j}}{d x}\right\|^{1 / 2}\left\|v^{j}\right\|^{1 / 2}+C_{2} \frac{\left\|v^{j}\right\|}{\beta_{n}^{1 / 2}},
$$


for some positives constants $C_{1}$ and $C_{2}$. This implies, using 3.22 , that $\frac{\left\|v^{j}\right\|_{\infty}}{\beta_{n}^{1 / 2}}$ is bounded.

Then, for every $k$ in $I_{\mathcal{M}}$, by $(3.25), q_{k, n}$ converge to zero and then $\beta_{n} p_{k, n}$ converge to zero in view of (3.24). In particular $p_{k, n}$ converge to zero.

Second step. We notice that from (2.4) we have

$$
\left\|\left(\mathbf{i} \beta_{n} I-\mathcal{A}\right) z_{n}\right\|_{H} \geq\left.\left|\operatorname{Re}\left\langle\mathbf{i} \beta_{n} I-\mathcal{A}\right) z_{n}, z_{n}\right\rangle_{H}\left|=\sum_{k \in I_{\mathcal{S}}}\right| v_{n}\left(a_{k}\right)\right|^{2} .
$$

Then, by $(3.21)$

$$
\left|v_{n}\left(a_{k}\right)\right| \longrightarrow 0, \forall k \in I_{\mathcal{S}}
$$

This further leads to

$$
\left|\beta_{n} y_{n}\left(a_{k}\right)\right| \longrightarrow 0, \forall k \in I_{\mathcal{S}} .
$$

due to 3.22 and the trace theorem.

We have also

$$
\frac{d y^{j_{k}}}{d x}\left(a_{k}\right) \longrightarrow 0, \quad \forall k \in I_{\mathcal{S}}
$$

Third step. Substituting (3.22) into (3.23) and (3.24) into 3.25) to get

$$
\begin{aligned}
& -\beta_{n}^{2} y_{n}^{j}-\frac{d^{2} y_{n}^{j}}{d x^{2}}=g_{n}^{j}+\mathbf{i} \beta_{n} f_{n}^{j}, \quad j \in J \\
& -\beta_{n}^{2} p_{k, n}+v_{n}\left(a_{k}\right)=r_{k, n}+\mathbf{i} \beta_{n} h_{k, n} .
\end{aligned}
$$

Next, we take the inner product of 3.29 with $b \frac{d y_{n}^{j}}{d x}$ in $L^{2}\left(0, \ell_{j}\right)$ for $b \in \mathcal{C}^{1}\left(\left[0, \ell_{j}\right]\right)$ we get

$$
\begin{aligned}
\frac{1}{2} \beta_{n}^{2}\left[\left|y_{n}^{j}(x)\right|^{2} b(x)\right]_{0}^{\ell_{j}}+\frac{1}{2}\left[\left|\frac{d y_{n}^{j}}{d x}(x)\right|^{2} b(x)\right]_{0}^{\ell_{j}} & +\left[\operatorname{Re}\left(\mathbf{i} \beta_{n} f_{n}^{j}(x) \overline{y_{n}^{j}}(x) b(x)\right)\right]_{0}^{\ell_{j}} \\
& -\frac{1}{2} \int_{0}^{\ell_{j}}\left(\left|\beta_{n} y_{n}^{j}\right|^{2}+\left|\frac{d y_{n}^{j}}{d x}\right|^{2}\right) \frac{d b}{d x} d x \longrightarrow 0 .
\end{aligned}
$$

With (3.27) and (3.28), this leads to

$$
\frac{1}{2} \int_{0}^{\ell_{j_{k}}}\left(\left|\beta_{n} y_{n}^{j_{k}}\right|^{2}+\left|\frac{d y_{n}^{j_{k}}}{d x}\right|^{2}\right) d x \longrightarrow 0
$$

for every $k$ in $I_{\mathcal{S}}$, by taking $b=x$ or $b=\ell_{j_{k}}-x$. Moreover, as in [15] it follows that

$$
\beta_{n} y_{n}\left(a_{s}\right) \longrightarrow 0, \frac{d y_{n}^{j_{k}}}{d x}\left(a_{s}\right) \longrightarrow 0, \text { and } \operatorname{Re}\left(i \beta_{n} f_{n}\left(a_{s}\right) \overline{y_{n}}\left(a_{s}\right)\right) \longrightarrow 0
$$

where $a_{s}$ is the end of $e_{j_{k}}$, different from $a_{k}$. We then conclude by iteration, that for every $j$ in $J$,

$$
\int_{0}^{\ell_{j}}\left(\left|\beta_{n} y_{n}^{j}\right|^{2}+\left|\partial_{x} y_{n}^{j}\right|^{2}\right) d x \longrightarrow 0
$$




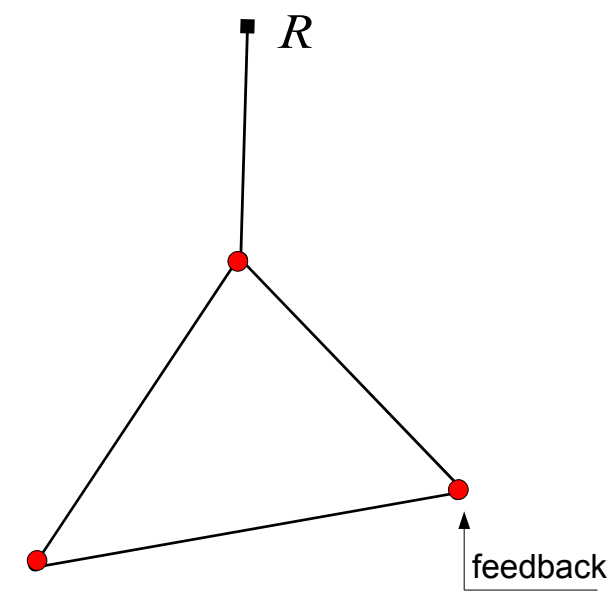

Figure 2. Circuit

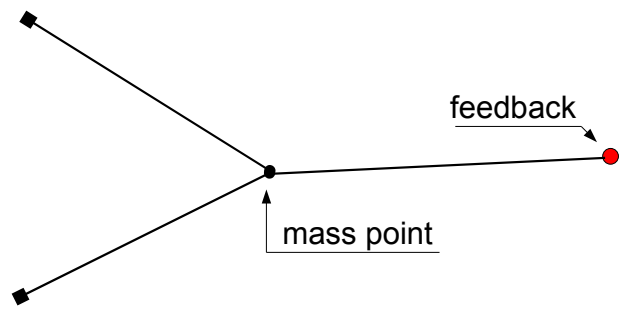

FiguRE 3. Start

Finally, in view of 3.22$)$, we also get

$$
\left\|v_{n}^{j}\right\| \longrightarrow 0, \text { for } j \in J \text {. }
$$

which implies that $\left\|z_{n}\right\|_{H} \longrightarrow 0$ : clearly contradicts 3.21$)$.

\section{Some other cases}

In this part we consider two particular cases. In the first, (Figure 2), there is a circuit in the graph. We prove that, even with much more controls, the exponential stability fails. The second case (Figure 3) proves that when we eliminate a control of a leaf in the initial case then the exponential stability fails.

\subsection{A circuit. (Figure 2)}

In this part we suppose that $\mathcal{T}$ contains a circuit (Figure 2 $)$, with feedbacks at each inner node. Then the second equation in (1.1) will be

$$
\sum_{j \in J_{k}} d_{k j} \partial_{x} y^{j}\left(a_{k}, t\right)=s_{1}^{\prime}(t)-y_{t}\left(a_{k}, t\right), \quad a_{k} \in I_{\mathcal{M}}
$$

and we can rewrite the system (1.1) in the Hilbert space $H$ as

$$
z^{\prime}(t)=\mathcal{A} z(t) .
$$

Except that, here, the first equation in 1.3 will be replaced by the following

$$
\sum_{j \in J_{k}} d_{k j} \frac{d y^{j}}{d x}\left(a_{k}\right)=q_{k}-y_{t}\left(a_{k}\right), \forall k \in I_{\mathcal{M}} .
$$


The operator $\mathcal{A}$ generates a $\mathcal{C}_{0}$-semigroup of contraction $(S(t))_{t \geq 0}$.

Next we suppose, without loss of generality, that $\ell_{1}=\ell_{2}=\ell_{3}=1$. We have the first result of asymptotic behavior

Theorem 4.1. $(S(t))_{t \leq 0}$ is asymptotically stable if and only if $\ell_{4}$ is irrational and not in $\pi \mathbb{Z}$.

Proof. First, if $\ell_{4}$ is in $\pi \mathbb{Z}$ then $\mathbf{i}$ is an eigenvalue of $\mathcal{A}$ (as in the case of a tree) and if $\ell_{4}=\frac{a}{b}$ with $a$ and $b$ integer, then $\mathbf{i} b \pi$ is an eigenvalue of $\mathcal{A}$.

Now, we suppose that $\ell_{4} \notin \mathbb{Q}$ and $\ell_{4} \notin \pi \mathbb{Z}$. We only need to prove that $\mathbf{i} \mathbb{R} \rho(\mathcal{A})$. For this, we will prove that the equation

$$
\mathcal{A} z=\mathbf{i} \beta z
$$

with $z=\left(\begin{array}{c}y \\ v \\ p \\ q\end{array}\right) \in \mathcal{D}(\mathcal{A})$ and $\beta \in \mathbb{R}$ has only trivial solution.

we can show that $v\left(a_{k}\right)=0$ for $k \in I_{\mathcal{M}}$. Now the equation (4.31) can be rewritten explicitly as

$$
\begin{aligned}
v^{j} & =\mathbf{i} \beta y^{j}, \quad j \in J, \\
\frac{d^{2} y^{j}}{d x^{2}} & =\mathbf{i} \beta v^{j}, \quad j \in J, \\
q_{k} & =\mathbf{i} \beta p_{k}, \quad k \in I_{\mathcal{M},} \\
-p_{k}-v\left(a_{k}\right) & =\mathbf{i} \beta q_{\kappa}, \quad k \in I_{\mathcal{M}} .
\end{aligned}
$$

If $\beta=0$ then as in for the initial example we show that $y=0$.

Next, we suppose that $\beta \neq 0$. We have $y\left(a_{k}\right)=0$ for every $k \in I_{\mathcal{M}}$

$$
\begin{aligned}
\beta^{2} y^{j}+\frac{d^{2} y^{j}}{d x^{2}} & =0, \quad j \in J, \\
\left(\beta^{2}-1\right) p_{k} & =0, \quad k \in I_{\mathcal{M}} .
\end{aligned}
$$

Then, since $y\left(a_{k}\right)=0$ for every $k$ then $y^{j}$ is of the form

$$
y^{j}=\alpha_{j} \sin (\beta x) .
$$

As in the case of a tree we consider two cases: $\beta^{2}=1$ and $\beta^{2} \neq 1$

First case: $\beta^{2}=1$. we have $\alpha_{1}=\alpha_{2}=\alpha_{3}=0$ and since $\ell_{4} \notin \pi \mathbb{Z}, \alpha_{4}=0$. Return back to the balance conditions and (4.33), at inner nodes, one can deduce that $q=p=0$ and hence $y=0$.

Second case: $\beta^{2} \neq 1$. we have $p_{k}=q_{k}=0$ for every $k$ in $I_{\mathcal{M}}$. Then $y=0$.

Theorem 4.2. The semigroup $(S(t))_{t \geq 0}$ is not exponentially stable, even if $\ell_{4}$ is irrational and not in $\pi \mathbb{Z}$.

Proof. To prove that $(S(t))_{t \geq 0}$ is not exponentially stable we consider the sequence $f_{n}$ of vectors of $H$ defined by $f_{n}=\left(0, g_{n}, 0,0\right)$ where $g_{n}=\left(0,-\sin \beta_{n} x, 0,0\right)$ and $\beta_{n}$ is 
a sequence of real numbers satisfying $\beta_{n} \longmapsto+\infty$ and will be defined later. We then prove that the sequence $z_{n}=\left(y_{n}, v_{n}, p_{n}, q_{n}\right)$ of elements of $\mathcal{D}(\mathcal{A})$ such that

$$
\left(\mathbf{i} \beta_{n}-\mathcal{A}\right) z_{n}=f_{n}
$$

is not bounded.

The sequence $y_{n}$ should satisfy

$$
\left\{\begin{array}{l}
\beta_{n}^{2} y_{n}^{j}+\partial_{x}^{2} y_{n}^{j}=0, \text { for } j=1,3,4 \\
\beta_{n}^{2} y_{n}^{2}+\partial_{x}^{2} y_{n}^{2}=\sin \beta_{n} x
\end{array}\right.
$$

Then, for $j=1,3,4$ there exists two complex numbers $a_{j}$ and $b_{j}$ such that

$$
\left\{\begin{array}{l}
y_{n}^{j}=a_{j} \sin \left(\beta_{n} x\right)+b_{j} \cos \left(\beta_{n} x\right) \\
\frac{d y_{n}^{j}}{d x}=-\beta_{n} b_{j} \sin \left(\beta_{n} x\right)+\beta_{n} a_{j} \cos \left(\beta_{n} x\right)
\end{array}\right.
$$

and there exists $a_{2}$ and $b_{2}$ in $\mathbb{C}$ such that

$$
\left\{\begin{array}{l}
y_{n}^{2}=a_{2} \sin \left(\beta_{n} x\right)+\left(-\frac{x}{2 \beta_{n}}+b_{2}\right) \cos \left(\beta_{n} x\right), \\
\frac{d y_{n}^{2}}{d x}=\left(\frac{x}{2}-\beta_{n} b_{2}\right) \sin \left(\beta_{n} x\right)+\left(-\frac{1}{2 \beta_{n}}+\beta_{n} a_{2}\right) \cos \left(\beta_{n} x\right) .
\end{array}\right.
$$

The boundary and transmission conditions are expressed as follows

$$
\left\{\begin{array}{l}
a_{1} \sin \left(\beta_{n}\right)+b_{1} \cos \left(\beta_{n}\right)=0 \\
b_{1}=b_{2}=b_{3} \\
-\frac{1}{2 \beta_{n}}+\beta_{n} a_{1}+\beta_{n} a_{2}+\beta_{n} a_{3}=-i \beta_{n} b_{1} \\
a_{2} \sin \left(\beta_{n}\right)+\left(-\frac{1}{2 \beta_{n}}+b_{2}\right) \cos \left(\beta_{n}\right)=b_{4} \\
\beta_{n} a_{4}-\left(\left(\frac{1}{2}-\beta_{n} b_{2}\right) \sin \left(\beta_{n}\right)+\left(-\frac{1}{2 \beta_{n}}+\beta_{n} a_{2}\right) \cos \left(\beta_{n}\right)\right)=-i \beta_{n} b_{4} \\
a_{3} \sin \left(\beta_{n}\right)+b_{1} \cos \left(\beta_{n}\right)=a_{4} \sin \left(\beta_{n} \ell_{4}\right)+b_{4} \cos \left(\beta_{n} \ell_{4}\right) \\
-\beta_{n} b_{3} \sin \left(\beta_{n}\right)+\beta_{n} a_{3} \cos \left(\beta_{n}\right)-\beta_{n} b_{4} \sin \left(\beta_{n} \ell_{4}\right)+\beta_{n} a_{4} \cos \left(\beta_{n} \ell_{4}\right)= \\
i \beta_{n}\left(a_{3} \sin \left(\beta_{n}\right)+b_{1} \cos \left(\beta_{n}\right)\right) .
\end{array}\right.
$$

Then

$$
\left\{\begin{array}{l}
a_{1} \sin \left(\beta_{n}\right)+b_{1} \cos \left(\beta_{n}\right)=0 \\
-\frac{1}{2 \beta_{n}}+\beta_{n} a_{1}+\beta_{n} a_{2}+\beta_{n} a_{3}=-i \beta_{n} b_{1} \\
a_{2} \sin \left(\beta_{n}\right)+\left(-\frac{1}{2 \beta_{n}}+b_{1}\right) \cos \left(\beta_{n}\right)=b_{4} \\
\beta_{n} a_{4}-\left(\left(\frac{1}{2}-\beta_{n} b_{1}\right) \sin \left(\beta_{n}\right)+\left(-\frac{1}{2 \beta_{n}}+\beta_{n} a_{2}\right) \cos \left(\beta_{n}\right)\right)=-i \beta_{n} b_{4} \\
a_{3} \sin \left(\beta_{n}\right)+b_{1} \cos \left(\beta_{n}\right)=a_{4} \sin \left(\beta_{n} \ell_{4}\right)+b_{4} \cos \left(\beta_{n} \ell_{4}\right) \\
\beta_{n} a_{3} e^{-\mathbf{i} \beta_{n}}-\beta_{n} b_{4} \sin \left(\beta_{n} \ell_{4}\right)+\beta_{n} a_{4} \cos \left(\beta_{n} \ell_{4}\right)=i \beta_{n} b_{1} e^{-\mathbf{i} \beta_{n}}
\end{array}\right.
$$

Hence

$$
(F B+A G) \beta_{n} b_{1}=A H-F \beta_{n} C
$$

where

$$
\begin{aligned}
A & =\left(1+\cos \left(\beta_{n} \ell_{4}\right)\right) \sin \left(\beta_{n}\right)+e^{-\mathbf{i} \beta_{n}} \sin \left(\beta_{n} \ell_{4}\right) \\
B & =\left(2-\cos \left(\beta_{n} \ell_{4}\right) \cos \left(\beta_{n}\right)+\mathbf{i}\left(e^{-\mathbf{i} \beta_{n}} \sin \left(\beta_{n} \ell_{4}\right)-\sin \left(\beta_{n}\right)\right)\right. \\
C & =\frac{1}{2 \beta_{n}^{2}} \sin \left(\beta_{n}\right)-\left(\frac{1}{2} \sin \left(\beta_{n}\right)+\frac{1}{2}(-1+\mathbf{i}) \cos \left(\beta_{n}\right)\right) \sin \left(\beta_{n} \ell_{4}\right) \\
& +\frac{1}{2 \beta_{n}} \cos \left(\beta_{n}\right) \cos \left(\beta_{n} \ell_{4}\right)
\end{aligned}
$$


and

$$
\begin{aligned}
F & =e^{-\mathbf{i} \beta_{n}}\left(\cos \beta_{n}-1\right)-\sin \left(\beta_{n}\right) \sin \left(\beta_{n} \ell_{4}\right), \\
G & =e^{-\mathbf{i} \beta_{n}}\left(\cot \beta_{n}-2 \mathbf{i}\right)-\cos \left(\beta_{n}\right) \sin \left(\beta_{n} \ell_{4}\right)-\mathbf{i} e^{-\mathbf{i} \beta_{n}} \cos \left(\beta_{n} \ell_{4}\right), \\
H & =-\frac{1}{2 \beta_{n}} e^{-\mathbf{i} \beta_{n}}-\frac{1}{2 \beta_{n}} \cos \left(\beta_{n}\right) \sin \left(\beta_{n} \ell_{4}\right)-\frac{1}{2} \sin \left(\beta_{n}\right) \cos \left(\beta_{n} \ell_{4}\right) \\
& -\frac{1}{2}(-1+\mathbf{i}) \cos \left(\beta_{n}\right) \cos \left(\beta_{n} \ell_{4}\right) .
\end{aligned}
$$

Now by using the Asymptotic Dirichlet's theorem [14, there exists $\left(p_{n}, q_{n}\right) \in \mathbb{N}^{2}$ such that $\frac{p_{n}}{q_{n}}$ converge to $\ell_{4}, p_{n}$ and $q_{n}$ tend to infinity as $n$ goes to infinity and for every $n$ in $\mathbb{N}$

$$
\left|q_{n} \ell_{4}-p_{n}\right|<\frac{1}{q_{n}}
$$

Take $\beta_{n}=2 \pi q_{n}+\frac{2 \pi}{q_{n}^{1 / 4}}$, then there exists a positive integer $n_{0}$ such that for every integer $n \geq n_{0}$,

$$
0<\lambda_{n}:=-\frac{2 \pi}{q_{n}}+\frac{2 \pi \ell_{4}}{q_{n}^{1 / 4}}<\beta_{n} \ell_{4}-2 \pi p_{n}<\mu_{n}:=\frac{2 \pi}{q_{n}}+\frac{2 \pi \ell_{4}}{q_{n}^{1 / 4}}<\frac{\pi}{2}
$$

and

$$
\sin \left(\lambda_{n}\right)<\sin \left(\beta_{n} \ell_{4}\right)<\sin \left(\mu_{n}\right), \quad \cos \left(\lambda_{n}\right)<\cos \left(\beta_{n} \ell_{4}\right)<\cos \left(\mu_{n}\right) .
$$

Moreover $\sin \left(\beta_{n} \ell_{4}\right)$ and $\cos \left(\beta_{n} \ell_{4}\right)$ satisfy the following asymptotic approximations,

$$
\sin \left(\beta_{n} \ell_{4}\right)=\frac{2 \pi \ell_{4}}{q_{n}^{1 / 4}}+o\left(\frac{1}{q_{n}^{1 / 4}}\right), \quad \cos \left(\beta_{n} \ell_{4}\right)=1+o\left(\frac{1}{q_{n}^{1 / 4}}\right) .
$$

We have also

$\sin \left(\beta_{n}\right)=\sin \left(\frac{2 \pi}{q_{n}^{1 / 4}}\right)=\frac{2 \pi}{q_{n}^{1 / 4}}+o\left(\frac{1}{q_{n}^{1 / 4}}\right), \cos \left(\beta_{n}\right)=1+o\left(\frac{1}{q_{n}^{1 / 4}}\right)$, and $\cot \left(\beta_{n}\right)=\frac{q_{n}^{1 / 4}}{2 \pi}\left(1+o\left(\frac{1}{q_{n}^{1 / 4}}\right)\right)$.

It follows that

$$
\begin{aligned}
& A=\frac{2 \pi\left(2+\ell_{4}\right)}{q_{n}^{1 / 4}}+o\left(\frac{1}{q_{n}^{1 / 4}}\right), \quad B=1+o(1), \quad C=(-1+\mathbf{i}) \frac{\pi \ell_{4}}{q_{n}^{1 / 4}}+o\left(\frac{1}{q_{n}^{1 / 4}}\right), \\
& F=-\frac{2 \pi^{2}\left(2 \ell_{4}+1\right)}{q_{n}^{1 / 2}}+o\left(\frac{1}{q_{n}^{1 / 2}}\right), \quad G=\frac{q_{n}^{1 / 4}}{2 \pi}-4 \mathbf{i}+o(1), \quad H=-\frac{1}{2}(-1+\mathbf{i})+o(1) .
\end{aligned}
$$

Return back to 4.36, we could write

$$
\left(2+\ell_{4}+o(1)\right) \beta_{n} b_{1}=o(1)-\beta_{n}\left(-(-1+\mathbf{i}) \frac{2 \pi^{3}\left(2 \ell_{4}+1\right) \ell_{4}}{q_{n}^{3 / 4}}+o\left(\frac{1}{q_{n}^{3 / 4}}\right)\right) \sim 2 \ell_{4}\left(2 \ell_{4}+1\right)(-1+\mathbf{i}) \pi^{3} q_{n}^{1 / 4} .
$$

Hence

$$
\beta_{n} b_{1} \sim 2 \ell_{4} \frac{2 \ell_{4}+1}{\ell_{4}+2}(-1+\mathbf{i}) \pi^{3} q_{n}^{1 / 4}
$$

Which implies that $\left\|y_{n}^{1}\right\|$ converges to infinity as $n$ goes to infinity and that consequently $z_{n}$ is not bounded.

Remark 4.3. A small change in the proof leads to the conclusion that a polynomial stability, can not be better than $\frac{1}{t^{2}}$ (by using a frequency domain characterization of polynomial stability of a $\mathcal{C}_{0}$-semigroup of contraction due to Borichev and Tomilov [6] ) Precisely we prove that the system is not $\frac{1}{t^{\alpha}}$-polynomially stable for every $\alpha$ in $(0,2)$. 
4.2. A start with two fixed endpoints. (Figure 3)

$$
\left\{\begin{array}{l}
y_{t t}^{j}-y_{x x}^{j}=0 \text { in }\left(0, \ell_{j}\right) \times(0, \infty), \quad j \in\{1,2,3\}, \\
\sum_{j=1}^{3} y_{x}^{j}(0, t)=s^{\prime}(t), s^{\prime \prime}(t)+s(t)=-y_{t}(0, t), \\
y^{j}(0, t)=y^{l}(0, t), \quad j, l \in\{1,2,3\}, \\
y^{2}(1, t)=y^{3}(1, t)=0, y_{x}^{1}(1, t)=-y_{t}(1, t), \\
s(0)=s_{0}, \quad s^{\prime}(0)=s_{1}, \\
y^{j}(x, 0)=y_{0}^{j}(x), y_{t}^{j}(x, 0)=y_{1}^{j}(x), \quad x \in(0,1), j \in\{1,2,3\} .
\end{array}\right.
$$

We can rewrite the system 4.37) in the Hilbert space $H$ as

$$
z^{\prime}(t)=\mathcal{A} z(t)
$$

where

$$
H=V \times \prod_{j=1}^{3} L^{2}\left(0, \ell_{3}\right) \times \mathbb{C}^{2}
$$

with

$$
V=\left\{\Phi \in \prod_{j \in J} H^{1}\left(0, \ell_{j}\right), \Phi^{2}\left(\ell_{2}\right)=\Phi^{3}\left(\ell_{3}\right)=0, \Phi^{j}(0)=\Phi^{l}(0), j, l \in\{1,2,3\}\right\} .
$$

and

with

$$
\mathcal{A}\left(\begin{array}{c}
y \\
v \\
p \\
q
\end{array}\right)=\left(\begin{array}{c}
v \\
\partial_{x}^{2} y \\
q \\
-p-v(0)
\end{array}\right), \forall\left(\begin{array}{l}
y \\
v \\
p \\
q
\end{array}\right) \in \mathcal{D}(\mathcal{A})
$$

$$
\begin{aligned}
\mathcal{D}(\mathcal{A})= & \left\{(y, v, p, q) \in\left[\prod_{j=1}^{3} H^{2}\left(0, \ell_{j}\right) \cap V\right] \times V \times \mathbb{C}^{2} ;\right. \\
& \left.\sum_{j=1}^{3} \frac{d y^{j}}{d x}(0)=q, \text { and } \frac{d y^{1}}{d x}\left(\ell_{1}\right)=-v^{1}\left(\ell_{1}\right)\right\} .
\end{aligned}
$$

The operator $\mathcal{A}$ generates a $\mathcal{C}_{0}$-semigroup of contraction $(S(t))_{t \geq 0}$.

If we take $\ell_{1}=\ell_{2}=1$ then we have the following result.

Theorem 4.4. the semigroup $(S(t))_{t \geq 0}$ is asymptotically stable if and only if $\ell_{3}$ irrational and not in $\pi \mathbb{Z}$.

Even if $\ell_{3}$ irrational and not in $\pi \mathbb{Z}$, the semigroup $(S(t))_{t \geq 0}$ is not exponentially stable.

Proof. As in the previous case, there exists $\left(p_{n}, q_{n}\right) \in \mathbb{N}^{2}$ such that $\frac{p_{n}}{q_{n}}$ converge to $\ell_{4}$ as $n$ goes to infinity. Then $q_{n}$ converge to infinity and there is a subsequence of $q_{n}$ denoted $q_{n}$ such that

$$
\left|q_{n} \ell_{3}-p_{n}\right|<\frac{1}{q_{n}}
$$


Then we take $\beta_{n}=2 \pi q_{n}+\frac{2 \pi}{q_{n}^{1 / 4}}$ and $f_{n}=\left(0, g_{n}, 0,0\right)$ with $g_{n}=\left(0,-\sin \beta_{n} x, 0\right)$. The sequence $\beta_{n}$ tends to infinity as $n$ goes to infinity, $f_{n} \in H$ and $f_{n}$ is bounded. To conclude, we prove as in the previous case that $z_{n}$ defined by $\left(\mathcal{A}-\mathbf{i} \beta_{n}\right) z_{n}=f_{n}$ is bounded.

\section{A chain with non equal mass points}

In this section, we consider a particular network which is a chain of $N$ edges $(N \geq 2)$ and $p=N+1$ vertices such that the $(N-1)$ interior vertices $a_{j}$ are point masses with mass $m_{j}$. But the masses $m_{j}$ are not necessery equal (Figure 4).

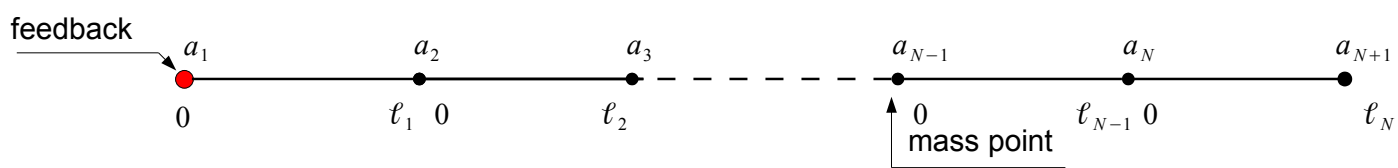

FiguRE 4. A chain of strings

Precisely, we consider the following system

$$
\left\{\begin{array}{l}
y_{t t}^{j}-y_{x x}^{j}=0 \text { in }\left(0, \ell_{j}\right) \times(0, \infty), \quad j \in\{1, \ldots, N\}, \\
y_{x}^{j}(0, t)-y_{x}^{j-1}\left(\ell_{j-1}, t\right)=s_{j}^{\prime}(t), \quad j \in\{2, \ldots, N\}, \\
m_{j} s_{j}^{\prime \prime}(t)+s_{j}(t)=-y_{t}(0, t), \quad j \in\{2, \ldots, N\}, \\
y^{j-1}\left(\ell_{j-1}, t\right)=y^{j}(0, t), \quad j \in\{2, \ldots, N\}, \\
y_{x}^{1}(0, t)=y_{t}(0, t), \\
y^{N}\left(\ell_{N}, t\right)=0, \\
s_{j}(0)=s_{j, 0}, \quad s_{j}^{\prime}(0)=s_{j, 1}, \quad j \in\{2, \ldots, N\}, \\
y^{j}(x, 0)=y_{0}^{j}(x), y_{t}^{j}(x, 0)=y_{k}^{1}(x), \quad x \in\left(0, \ell_{j}\right), j \in\{1, \ldots, N\} .
\end{array}\right.
$$

Note that the feedback is applied at the vertex $a_{1}$. We give a necessary and sufficient condition for the exponential stability of system (5.38). The general case of a tree with distinct masses at inner nodes is complicated for the moment.

To start we quickly redefine the associated state space $H$ and the operator $\mathcal{A}$ as follow:

$$
H=V \times \prod_{j=1}^{N} L^{2}\left(0, \ell_{j}\right) \times\left(\prod_{j=1}^{N-1} \mathbb{C}\right)^{2}
$$

with

$$
V=\left\{\Phi \in \prod_{j=1}^{N} H^{1}\left(0, \ell_{j}\right), \Phi^{N}\left(\ell_{N}\right)=0, \Phi^{j-1}\left(\ell_{j-1}\right)=\Phi^{j}(0), j=2, \ldots, N\right\} .
$$


and

$$
\mathcal{A}\left(\begin{array}{c}
y \\
v \\
p \\
q
\end{array}\right)=\left(\begin{array}{c}
v \\
\frac{d^{2} y}{d x^{2}} \\
q \\
-m^{-1}\left(p+v_{\mathcal{M}}\right)
\end{array}\right), \forall\left(\begin{array}{c}
y \\
v \\
p \\
q
\end{array}\right) \in \mathcal{D}(\mathcal{A}),
$$

with $-m^{-1}\left(p+v_{\mathcal{M}}\right)=\left(-\frac{1}{m j} p_{j}-\frac{1}{m_{j}} v\left(\ell_{j}\right)\right)_{j \in\{2, \ldots, N-1\}}$ and

$$
\left.\mathcal{D}(\mathcal{A})=\left\{(y, v, p, q) \in\left[\prod_{j \in J} H^{2}\left(0, \ell_{j}\right) \cap V\right] \times V \times \prod_{k \in I_{\mathcal{M}}} \mathbb{C}^{2} \text { satisfying } 5.39\right)\right\}
$$

where

$$
\left\{\begin{array}{l}
\frac{d y^{j}}{d x}(0, t)-\frac{d y^{j-1}}{d x}\left(\ell_{j-1}\right)=q_{j}, \quad j \in\{2, \ldots, N\} \\
\frac{d y^{1}}{d x}(0)=v^{1}(0) .
\end{array}\right.
$$


$\sigma(\mathcal{A})=\sigma_{p}(\mathcal{A})$

For every mass point $m$ we denote by $i_{1}(m), \ldots, i_{k_{m}}(m)$ the indices of the interior nodes with masses equal to $m$ and ordered as follow, $i_{1}(m)<i_{2}(m)<\ldots<i_{k_{m}}(m)$.

For $r=r(m) \in\left\{1, \ldots, k_{m}\right\}$ we define the scalars

$$
\begin{gathered}
\Pi_{m, r(m), s}= \\
\sum_{i_{r}=j_{0}<j_{1}<\cdots<j_{s}<i_{r+1}}\left(\prod_{i=0}^{s-1} c_{j_{i+1}} \sin \left(\beta \ell_{j_{i}}+\cdots+\beta \ell_{j_{i+1}-1}\right)\right) \sin \left(\beta \ell_{j_{s}}+\cdots+\beta \ell_{i_{r+1}-1}\right)
\end{gathered}
$$

and

$$
\begin{aligned}
\Delta_{r(m)} & :=(-1)^{i_{r+1}-i_{r}} \sin \left(\beta \ell_{i_{r}}+\ldots+\beta \ell_{i_{r+1}-1}\right) \\
& +\sum_{s=0}^{i_{r+1}-i_{r}-1}(-1)^{i_{r+1}-i_{r}+s} \Pi_{m, r(m), s},
\end{aligned}
$$

with, $\Delta_{r(m)}=\sin \left(\beta \ell_{r}\right)$ if $k_{m}=1$.

Then we have the following result of asymptotic behavior of the system (5.38):

Lemma 5.1. The system defined by $(5.38)$ is exponentially stable if and only if for every mass point $m$ and for every $r(m), \Delta_{r(m)} \neq 0$.

Proof. The first question is whether $\mathbf{i} \mathbb{R}$ belongs to $\rho(A)$. Thus, we will solve the equation

$$
\mathcal{A} z=\mathbf{i} \beta z
$$

of unknown $z=\left(\begin{array}{c}y \\ v \\ p \\ q\end{array}\right) \in \mathcal{D}(\mathcal{A})-\{0\}$ and $\beta \in \mathbb{R}$. 
If $\beta=0$ then $z=0$. Thus suppose that $\beta \neq 0$. We have

$$
\begin{aligned}
v^{j} & =\mathbf{i} \beta y^{j}, \quad \beta^{2} y^{j}+\frac{d^{2} y^{j}}{d x^{2}}=0, \quad j \in\{1, \ldots, N\}, \\
q^{j} & =\mathbf{i} \beta p^{j}, \quad\left(m_{j} \beta^{2}-1\right) p_{j}=v\left(a_{j}\right), \quad j \in\{2, \ldots, N\} .
\end{aligned}
$$

The function $y^{j}, j \in\{1, \ldots, N\}$ is then of the form

$$
y^{j}=\alpha_{j} \cos (\beta x)+\gamma_{j} \sin (\beta x)
$$

If $m_{j} \beta^{2} \neq 1$ for every $j$ in $\{2, \ldots, N\}$ then we prove by iteration, starting with $j=1$, that $y^{j}=0$ for every $j$ in $\{2, \ldots, N\}$ and consequently $z=0$.

Now we suppose the existence of a mass point $m$ with $m \beta^{2}=1$, Let $i_{1}(m)<\ldots<$ $i_{k_{m}}(m)$ the indices of inner nodes with masses equal to $m$. Then as in the first case $y^{j}=0$ for every $j<i_{1}$. Let $r=r(m) \in\left\{1, \ldots, k_{m}\right\}$ we have the following system

$$
\left\{\begin{array}{l}
y^{r}(0)=0 \\
\text { for } j=i_{r} \text { to } j=i_{r+1}-2, y^{j}\left(\ell_{j}\right)=y^{j+1}(0) \\
\text { and }-\frac{d y^{j}}{d x}\left(\ell_{j}\right)+\frac{d y^{j+1}}{d x}(0)=q^{j+1} \\
y^{i_{r+1}-1}\left(\ell_{i_{r+1}-1}\right)=0
\end{array}\right.
$$

It is obvious that the system (5.40) has trivial solution if and only if for every $m$ and every $r(m)$ the system 5.43 has a trivial solution.

By changes of indices we can suppose that $i_{r}=2, i_{r+1}=N+1$. The system (5.43) can be rewritten as

$$
\left\{\begin{array}{l}
\gamma_{2}=0 \\
\text { for } j=2 \text { to } j=N-1, \alpha_{j} \cos \left(\beta \ell_{j}\right)+\gamma_{j} \sin \left(\beta \ell_{j}\right)=\gamma_{j+1} \\
\text { and } \alpha_{j} \sin \left(\beta \ell_{j}\right)-\gamma_{j} \cos \left(\beta \ell_{j}\right)+\frac{1}{\beta} \frac{1}{m_{j}-m} \alpha_{j+1}+\gamma_{j+1}=0 \\
\alpha_{N} \cos \left(\beta \ell_{N}\right)+\gamma \sin \left(\beta \ell_{N}\right)=0
\end{array}\right.
$$

The matrix of such system is

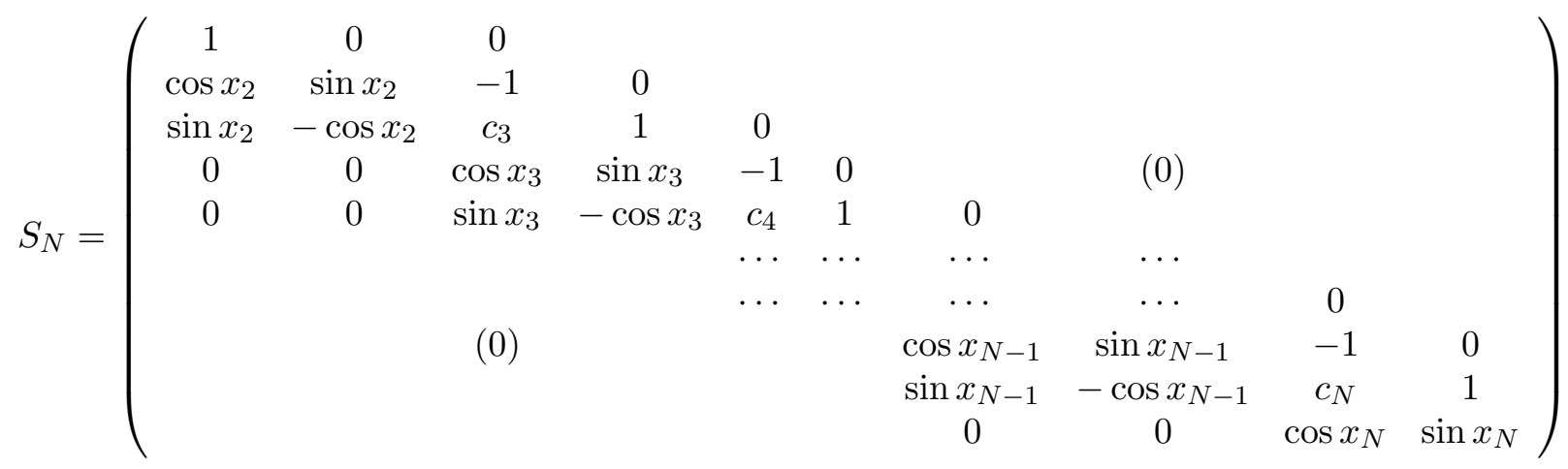

where $x_{j}=\beta \ell_{j}$ and $c_{j}=\frac{1}{\beta\left(m_{j}-m\right)}$. We want to calculate the determinant $\Delta_{N}$ of $S_{N}$ For this, Let $M_{N}$ the determinant of the matrix obtain from $S_{N}$ by replacing $\cos x_{N}$ and $\sin x_{N}$ in the last line by $\sin x_{N}$ and $-\cos x_{N}$ respectively. 
One can verifies easily that

$$
\begin{aligned}
\Delta_{2} & =\sin x_{2}, \quad \Delta_{3}=-\sin \left(x_{2}+x_{3}\right)+c_{3} \sin x_{2} \sin x_{3}, \\
\Delta_{4} & =\sin \left(x_{2}+x_{3}+x_{4}\right)-c_{3} \sin x_{2} \sin \left(x_{3}+x_{4}\right)-c_{4} \sin \left(x_{2}+x_{3}\right) \sin x_{4} \\
& +c_{3} c_{4} \sin x_{2} \sin x_{3} \sin x_{4}, \\
M_{2} & =-\cos x_{2}, \quad M_{3}=\cos \left(x_{2}+x_{3}\right)-c_{3} \sin x_{2} \cos x_{3}, \\
M_{4} & =-\cos \left(x_{2}+x_{3}+x_{4}\right)+c_{3} \sin x_{2} \cos \left(x_{3}+x_{4}\right)+c_{4} \sin \left(x_{2}+x_{3}\right) \cos x_{4} \\
& -c_{3} c_{4} \sin x_{2} \sin x_{3} \cos x_{4} .
\end{aligned}
$$

We will prove by induction that for every integer $N \geq 2$,

$$
\begin{aligned}
& \Delta_{N}=(-1)^{N} \sin \left(x_{2}+\ldots+x_{N}\right)+(-1)^{N-1} \sum_{j=2}^{N-1} c_{j+1} \sin \left(x_{2}+\ldots+x_{j-1}\right) \sin \left(x_{j}+\ldots+x_{N}\right) \\
& +5.44) \\
& +\sum_{s=2}^{N-2}(-1)^{N-1-s} \sum_{2=j_{0}<j_{1}<\cdots<j_{s} \leq N}\left(\prod_{i=0}^{s-1} c_{j_{i+1}} \sin \left(x_{j_{i}}+\ldots+x_{j_{i+1}-1}\right)\right) \sin \left(\beta \ell_{j_{s}}+\ldots+\beta \ell_{N}\right)
\end{aligned}
$$

and

$$
\begin{aligned}
& M_{N}=(-1)^{N+1} \cos \left(x_{2}+\ldots+x_{N}\right)+(-1)^{N} \sum_{j=2}^{N-1} c_{j+1} \sin \left(x_{2}+\ldots+x_{j-1}\right) \cos \left(x_{j}+\ldots+x_{N}\right) \\
& \quad+5.45) \\
& +\sum_{s=2}^{N-2}(-1)^{N-s} \sum_{2=j_{0}<j_{1}<\cdots<j_{s} \leq N}\left(\prod_{i=0}^{s-1} c_{j+1} \sin \left(x_{j_{i}}+\ldots+x_{j_{i+1}-1}\right)\right) \cos \left(x_{j_{s}}+\ldots+x_{N}\right) .
\end{aligned}
$$

Such rules are true for $N=2$. and $N=3$ let $N \in \mathbb{N}$ with $N \geq 3$ and suppose that (5.44) and (5.45) are true. Some calculations leads to

$$
\begin{aligned}
& \Delta_{N}=\left(-\cos x_{N}+c_{N} \sin x_{N}\right) \Delta_{N-1}+\left(\sin x_{N}\right) M_{N-1}, \\
& M_{N}=\left(-\sin x_{N}-c_{N} \cos x_{N}\right) \Delta_{N-1}-\left(\cos x_{N}\right) M_{N-1}
\end{aligned}
$$

We can now verify the rule $(5.44)$ to order $N$ :

$$
\begin{aligned}
& \Delta_{N} \\
= & \left((-1)^{N} \sin \left(x_{2}+\ldots+x_{N}\right)+(-1)^{N-1} \sum_{j=2}^{N-1} c_{j+1} \sin \left(x_{2}+\ldots+x_{j-1}\right) \sin \left(x_{j}+\ldots+x_{N}\right)\right. \\
& \left.+\sum_{s=2}^{N-3}(-1)^{N-1-s} \sum_{2=j_{0} \leq j_{1}<\ldots<j_{s} \leq N-1}\left(\prod_{i=0}^{s-1} c_{j_{i+1}} \sin \left(x_{j_{i}}+\ldots+x_{j_{i+1}-1}\right)\right) \sin \left(x_{j_{s}}+\ldots+x_{N}\right)\right) \\
& \left.+\sum_{s=2}^{N-3}(-1)^{N-2-s} \sum_{2=j_{0} \leq j_{1}<\ldots<j_{s} \leq N-1} c_{N}\left(\prod_{i=0}^{s-1} c_{j_{i+1}} \sin \left(x_{j_{i}}+\ldots+x_{j_{i+1}-1}\right)\right) \sin \left(x_{j_{s}}+\ldots+x_{N-1}\right) \sin x_{N}\right) \\
= & \left((-1)^{N} \sin \left(x_{2}+\ldots+x_{N}\right)+(-1)^{N-1} \sum_{j=2}^{N-1} c_{j+1} \sin \left(x_{2}+\ldots+x_{j-1}\right) \sin \left(x_{j}+\ldots+x_{N}\right)\right. \\
& \left.+\sum_{s=2}^{N-2}(-1)^{N-1-s} \sum_{2=j_{0} \leq j_{1}<\cdots<j_{s} \leq N}\left(\prod_{i=0}^{s-1} c_{j_{i+1}} \sin \left(x_{j_{i}}+\ldots+x_{j_{i+1}-1}\right)\right) \sin \left(x_{j_{s}}+\ldots+x_{N}\right)\right) .
\end{aligned}
$$

A similar calculus, using (5.45) proves that $(5.45)$ is verified in order $N$.

We can now state the following results: 
The associated semigroup $S(t)$ is asymptotically stable if and only if $\Delta_{r(m)}$ is different from zero for every mass point $m$ and every $r(m)$. To conclude that $(S(t))_{t \geq 0}$ is exponentially stable, it suffices to prove that 3.20 is satisfied by $(S(t))_{t \geq 0}$, exactly as in the proof of Theorem 3.2

\section{Acknowledgment}

This work was carried out under Airbus Chair Grant. Financial support from Airbus Chair Grant is gratefully acknowledged.

\section{References}

[1] A.-B. Abdallah and F. Shel, Exponential Stability of a General Network of 1-d Thermoelastic Materials, Mathematical Control and Related Fields, 2, (2012), 1-16.

[2] K. Ammari, A. Henrot and M. Tucsnak, Asymptotic Behaviour of the solutions and optimal location of the actuator for the pointwise stabilization of a string, Asymptotic Analysis, 28 (2001), 215-240.

[3] K. Ammari and M. Jellouli, Stabilization of star-shaped networks of strings, Differential and Integral Equations, 17 (2004), 1395-1410.

[4] K. Ammari, M. Jellouli and M. Khenissi, Stabilization of generic trees of strings, J. Dynamical. Control. Systems, 11 (2005), 177-193.

[5] K. Ammari and S. Nicaise, Stabilization of elastic systems by collocated feedback, 2124, Springer, Cham, 2015.

[6] A. Boritchev and Y. Tomilov, Optimal polynomial decay of functions and operator semigroups, Math. Ann., 347 (2010), 455-478.

[7] H. Brezis, Analyse Fonctionnelle, Théorie et Applications, Masson, Paris, 1983.

[8] R. Dàger and E. Zuazua, Wave propagation, observation and control in 1-d flexible multi-structures, Springer-Verlag, Berlin, Mathématiques \& Applications, 2006.

[9] S. Ervedoza and M. Vanninathan, Controllability of a simplified model of fluid-structure interaction, COCV., 20 (2014), 547-575.

[10] F.-L. Huang, Characteristic condition for exponential stability of linear dynamical systems in Hilbert spaces, Ann. Diff. Eqs., 1 (1985), 43-56.

[11] Y. Liu, T. Takahashi and M. Tucsnak, Single input of a simplified fluid-structure interaction model, ESAIM Control Optim. Calc. Var., 19 (2013), 20-42.

[12] D. Mercier and V. Régnier, Spectrum of a Network of Euler-Bernoulli Beams, J. Math. Anal. Appl., 342 (2008), 174-196.

[13] J. Prüss, On the spectrum of $\mathcal{C}_{0}$-semigroups, Trans. Amer. Math. Soc., 284 (1984), 847-857.

[14] W.-M. Schmidt, Diophantine approximation, Lecture Notes in Mathematics, 785, Springer-Verlag, Berlin-Heidelberg-New York, 1980.

[15] F. Shel, Exponential Stability of a Network of Beams, J. Dynam. Control Syst., 21 (2015), 443-460.

[16] M. Tucsnak and M. Vanninathan, Locally distributed control for a model of fluid-structure interaction, System and Control Letters, 58 (2009), 547-552.

[17] J.-L. Vazquez and E. Zuazua, Large time behavior for a simplified 1D model of fluid-solid interaction, Comm. Partial Differential Equations, 28 (2003), 1705-1738.

[18] L Lack of collision in a simplified 1D model for fluid-solid interaction, Math. Models Methods Appl. Sci., 16 (2006), 637-678. 
UR Analysis and Control of Pde, UR 13ES64, Department of Mathematics, Faculty of Sciences of Monastir, University of Monastir, Tunisia

E-mail address: kais.ammari@fsm.rnu.tn

UR Analysis and Control of Pde, UR 13ES64, Department of Mathematics, Faculty of Sciences of Monastir, University of Monastir, Tunisia

E-mail address: farhat.shel@ipeit.rnu.tn

TIFR-CAM, Post Bag 6503, Bangalore 560065, India

E-mail address: vanni@math.tifrbng.res.in 